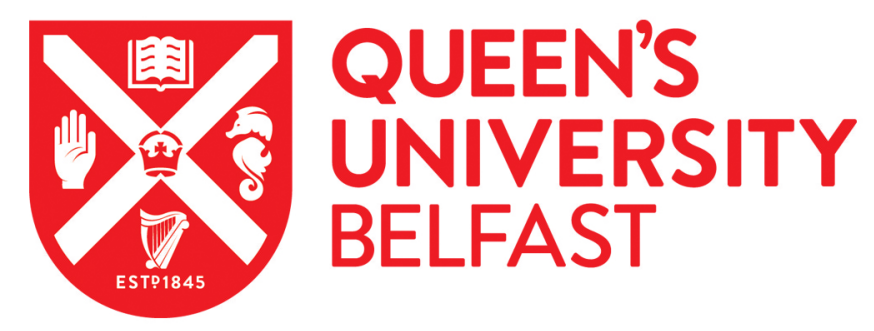

\title{
Efficient Incorporation of the RNS DataPath in Reverse Converter
}

Taheri, M., Sabbagh Molahosseini, A., \& Navi, K. (2021). Efficient Incorporation of the RNS DataPath in Reverse Converter. IEEE Transactions on Circuits and Systems II: Express Briefs, 68(4), 1388-1392. [9226450]. https://doi.org/10.1109/TCSII.2020.3031863

\section{Published in:}

IEEE Transactions on Circuits and Systems II: Express Briefs

\section{Document Version:}

Peer reviewed version

\section{Queen's University Belfast - Research Portal:}

Link to publication record in Queen's University Belfast Research Portal

\section{Publisher rights}

Copyright 2020 IEEE

This work is made available online in accordance with the publisher's policies. Please refer to any applicable terms of use of the publisher.

\section{General rights}

Copyright for the publications made accessible via the Queen's University Belfast Research Portal is retained by the author(s) and / or other copyright owners and it is a condition of accessing these publications that users recognise and abide by the legal requirements associated with these rights.

Take down policy

The Research Portal is Queen's institutional repository that provides access to Queen's research output. Every effort has been made to ensure that content in the Research Portal does not infringe any person's rights, or applicable UK laws. If you discover content in the Research Portal that you believe breaches copyright or violates any law, please contact openaccess@qub.ac.uk. 


\title{
Efficient Incorporation of the RNS Datapath in Reverse Converter
}

\author{
MohammadReza Taheri, Amir Sabbagh Molahosseini, Senior Member, IEEE, and Keivan Navi, \\ Senior Member, IEEE
}

\begin{abstract}
The class of moduli sets in the form of $\left\{2^{k}, 2^{n}-1,2^{n}+1\right.$, $\left.m_{4}\right\}$ with $m_{4} \in\left\{2^{r}+1,2^{r}-1\right\}$ has earned significant popularity in the implementation of the Residue Number System (RNS)-based computational systems, mainly thanks to the efficient arithmetic unit and a high degree of parallelism. However, its complicated inter-modulo computation leads to a high overhead associated with the complex reverse converter. This overhead is the main barrier for energy-efficient implementation of RNS-based devices, particularly for edge computing applications. This brief presents a new approach that embeds the reverse converter into the arithmetic unit of the RNS processor for the aforesaid well-known class of moduli sets. The effective hardware reuse in the proposed approach leads to an area and energy-efficient RNS realization for this class of moduli set. The experimental results based on $65 \mathrm{~nm}$ CMOS technology indicate the superiority of RNS realization by employing the proposed design methodology. The proposed architecture for a given RNS provides a substantial $17.4 \%$ areasaving and $13.32 \%$ less power-consumption on average compared to the traditional design approach, with the negligible penalty in delay.
\end{abstract}

Index Terms-Arithmetic unit, modular arithmetic, parallel prefix adder, residue number system, reverse converter.

\section{INTRODUCTION}

$\mathrm{T}$ he inherently parallel and modular arithmetic properties of the Residue Number System (RNS) offer great advantages in the realization of application-specific digital signal processors (DSP) [1], [2]. Besides adding values to DSPs, RNS is also achieved growing popularity in the implementation of emerging applications like modern cryptosystems [3], highefficiency video coding [4], deep neural networks [5], and inmemory processing [6]. Enhancing the performance, cutting down the power consumption, and improving the reliability are certain critical constraints that stand RNS as a viable candidate for integrating the aforesaid computation-intensive applications in embedded systems and Internet-of-Things devices. For a more effective RNS-based implementation of such these application with an appropriate dynamic range, as well as good enough parallelism, two main categories, namely Class-I and Class-II, have gained more popularity [7]. The common property of Class-I is that one modulus is power-of-two, while

MR. Taheri and K. Navi are with the Department of Computer Science and Engineering, Shahid Beheshti University, G. C., Tehran 1983969411, Iran (email:moh_taheri@sbu.ac.ir; navi@sbu.ac.ir). the product of the other moduli is a Mersenne number. The second class is considered as a horizontal extension of a special type of Class-I three-moduli set with an additional modulus $m_{4}$ $\left(\left\{2^{k}, 2^{n}-1,2^{n}+1, m_{4}\right\}\right)$. It is worth mentioning here that the product of all odd moduli in Class-II RNS is not a Mersenne number.

Along with the great advantages, the reverse converter imposes a complex architecture due to the use of inter-modular computations. The majority of state-of-the-art works on the use of RNS for different applications have considered the reverse converter and modular arithmetic channels as two separate parts that result in high-area overhead [8]. Given the fact that during the reverse conversion phase, the datapath is unused, a recent study [9] revealed that the embedding reverse converter into the datapath of RNS leads to reduce the transcoder overhead. However, the presented method in [9] just covers RNS with the Class-I moduli sets, which has a very simple reverse converter block compare to the Class-II RNS. The striking features of Class-II RNS have attracted the attention of researchers toward the efficient development of its hardware component [8]. Due to the substantial hardware cost of the reverse conversion process of the Class-II RNS, looking for a solution which provides considerable hardware saving compared with previously reported solution, seems worthy. This brief is distinctive compared to the existing literature in that we will provide the first solution toward incorporation of the RNS datapath in the reverse converter for the Class-II moduli sets.

The rest of the brief is organized as follows. Section II briefly covers RNS background material and introduces the adopted notational convention. Section III presented the algorithm and the hardware structure of the proposed approach. Section IV evaluates the proposed approach against related works. Finally, Section V concludes the brief.

\section{BACKGROUND MATERIAL}

For the RNS $\left\{m_{1}, m_{2}, \ldots, m_{b}\right\}$ the weighted integer $X$ can be obtained from its corresponding residues $\left(x_{1}, x_{2}, \ldots, x_{b}\right)$ via new Chinese Remainder Theorem (CRT-I), and Mixed Radix Conversion (MRC) [8], [10]. Based on the CRT-I, the integer $X$ from its corresponding residues can be given by:

A. S. Molahosseini is with the School of Electronics, Electrical Engineering, and Computer Science, Queen's University Belfast, Belfast BT71NN, UK (email: a.sabbaghmolahosseini@qub.ac.uk). 


$$
X=x_{1}+m_{1}\left\langle k_{1}\left(x_{2}-x_{1}\right)+\sum_{i=2}^{b-1}\left(k_{i} \prod_{j=2}^{i}\left(m_{i}\left(x_{i+1}-x_{i}\right)\right)\right)\right\rangle_{N_{1}}
$$

where $N_{1}=M / m_{1}$, and $k_{i}$ is the multiplicative inverse of $\prod_{j=1}^{i} m_{j}$ modulo $\prod_{j=i+1}^{b} m_{j} . X$ also can be yielded from its residues by adopting the MRC as:

$$
X=\sum_{i=2}^{b}\left(v_{i}\left(\prod_{j=1}^{i-1} m_{j}\right)\right)+v_{1}
$$

where the mixed-radix coefficients $v_{i}$ are derived consecutively using the following recurrence relations:

$$
\begin{aligned}
& v_{i}=\langle F(i, i)\rangle_{m_{i}} ; \\
& F(i, j)=\left\{\begin{array}{cc}
x_{j} & i=1 \\
\left(F(i-1, j)-v_{i-1}\right)\left\langle m_{i-1}^{-1}\right\rangle_{m_{j}} & i>1
\end{array}\right.
\end{aligned}
$$

where $\left\langle m_{a}^{-1}\right\rangle_{m_{b}}$ is the multiplicative inverse of $m_{a}$ modulo $m_{b}$. The following notational convention is adopted throughout the rest of this brief.

- $A_{(i: j)}$ refers to an $(i-j+1)$-bit slice of $A$ from position $i$ to position $j$ such that $A_{(i: j)}=A_{(i)} A_{(i-1)} \ldots A_{(j+1)} A_{(j)}$.

- $\|$ denotes the operator which concatenates the binary representation of two integers, and $\Delta_{i}^{j}$ refers to a vector that is expanded by $j$ concatenated $i$ strings.

- $m_{i \leftrightarrow l}(i<l)$ refers to $\prod_{j=i}^{l} m_{j}$, and $X_{i \leftrightarrow l}=X \bmod m_{i \leftrightarrow l}$.

\section{INTEGRATING THE REVERSE CONVERTER INTO THE ARITHMETIC CHANNELS}

\section{A. General Formulation for Class-II RNS Reverse Converters}

In the following, we derive general formulations for the reverse converter of Class-II RNS. This class of moduli sets can be expressed in two composite-forms (CFs): $\left\{2^{k}\left(2^{2 n}-1\right), m_{4}\right\}$ as the CF-I, and $\left\{2^{k} m_{4}, 2^{2 n}-1\right\}$ as the CF-II, with $m_{4} \in\left\{2^{r}+1,2^{r}-1\right\}$. Let $\mathfrak{B}$ be a CF of RNS basis $B$. The number of elements of $\mathfrak{B}$ is less than or equal to that of $B$, and the product of all moduli of $\mathfrak{B}$ equals to the product to all moduli of $B$. The reverse converter for the CF-I can be obtained in two levels of design. The first level employs the CRT-I to obtain the intermediate number $X_{1 \leftrightarrow 3}$ as follows[7]:

$$
X_{1 \leftrightarrow 3}=x_{1}+2^{k}\left\langle S_{Y}+C_{Y}\right\rangle_{2^{2 n}-1}=x_{1}+2^{k} Y
$$

In the second level of design, the weighted number $X$ can be extracted by employing MRC for set $\left\{m_{1 \leftrightarrow 3}, m_{4}\right\}$ as:

$$
\begin{aligned}
X & =X_{1 \leftrightarrow 3}+m_{1 \leftrightarrow 3}\left\langle\left\langle m_{1 \leftrightarrow 3}^{-1}\right\rangle_{m_{4}}\left(x_{4}-X_{1 \leftrightarrow 3}\right)\right\rangle_{m_{4}} \\
& =x_{1}+2^{k} Y+m_{1 \leftrightarrow 3}\left\langle S_{L}+C_{L}\right\rangle_{m_{4}}=x_{1}+2^{k}\left(Y+\left(2^{2 n}-1\right) L\right) \\
& =(L \| Y-L)\left\|x_{1}=\left(L\left\|Y+\Delta_{1}^{2 n}\right\| \bar{L}+1\right)\right\| x_{1}
\end{aligned}
$$

The reverse converter for the CF-II can be expressed with a divide and conquer approach using MRC. To this, in the first step $X_{1,4}$ and $X_{2,3}$ are obtained from two moduli subsets $\left\{2^{k}, m_{4}\right\}$ and $\left\{2^{n}-1,2^{n}+1\right\}$, respectively:

$$
\begin{aligned}
& X_{1,4}=x_{1}+2^{k}\left\langle S_{I}+C_{I}\right\rangle_{m_{4}}=x_{1}+2^{k} I \\
& X_{2,3}=x_{3}+\left(2^{n}+1\right)\left\langle 2^{n-1}\left(x_{2}-x_{3}\right)\right\rangle_{2^{n}-1}
\end{aligned}
$$

The weighted number $X$ can be obtained considering moduli set $\left\{m_{1,4}, m_{2,3}\right\}$, and its corresponding residues $X_{1,4}$ and $X_{2,3}$ as follows:

$$
X=X_{1,4}+2^{k} m_{4}\left\langle\left\langle\left(2^{k} m_{4}\right)^{-1}\right\rangle_{2^{2 n}-1}\left(X_{2,3}-X_{1,4}\right)\right\rangle_{2^{2 n}-1}
$$

Because $X_{2,3}<2^{2 n}-1$ and $\langle\xi \times \delta\rangle_{\xi \times \sigma}=\xi \times\langle\delta\rangle_{\sigma}$, to avoid the utilization of extra adder in modulo $2^{n}-1, X_{2,3}$ can be reformulated as:

$$
X_{2,3}=\left\langle x_{3}+\left(2^{n}+1\right) 2^{n-1}\left(x_{2}-x_{3}\right)\right\rangle_{2^{2 n}-1}=\left\langle S_{Z}+C_{Z}\right\rangle_{2^{2 n}-1}
$$

with regards to (9), $X_{2,3}$ can be employed in (8) in carry-save form $S_{z}+C_{z}$ as follows.

$$
\begin{aligned}
X & =X_{1,4}+2^{k} m_{4}\left\langle\left\langle\left(2^{k} m_{4}\right)^{-1}\right\rangle_{2^{2 n}-1}\left(S_{Z}+C_{Z}-X_{1,4}\right)\right\rangle_{2^{2 n}-1} \\
& =x_{1}+2^{k} I+2^{k} m_{4}\left\langle S_{T}+C_{T}\right\rangle_{2^{2 n}-1}=x_{1}+2^{k}\left(I+m_{4} T\right)
\end{aligned}
$$

The simplification of (10) differs according to the value of $m_{4}$. For $m_{4}=2^{r}-1$, the following holds:

$$
\begin{aligned}
X & =x_{1}+2^{k}\left(I+\left(2^{r}-1\right) T\right)=(T \| I-T) \| x_{1} \\
& =\left(T\left\|I+\Delta_{1}^{r}\right\| \bar{T}+1\right) \| x_{1}
\end{aligned}
$$

for $m_{4}=2^{r}+1$, considering the fact when $I_{(r)}=1$ all the remaining bits of $I$ equal to 0 (i.e. $\left.I_{(r-1: 0)}=0\right)$, (10) can be simplified as follow:

$$
\begin{aligned}
X & =x_{1}+2^{k}\left(I+\left(2^{r}+1\right) T\right)=\left(2^{r} I_{(r)}+I_{(r-1: 0)}+\left(2^{r}+1\right) T\right) \| x_{1} \\
& =\left(T \| I_{(r-1: 0)}+T+\left(2^{r}-1\right) I_{(r)}+I_{(r)}\right) \| x_{1} \\
& =\left(T \| \Gamma+T+I_{(r)}\right) \| x_{1}
\end{aligned}
$$

Such that $\Gamma_{(i)}=I_{(i)} \vee I_{(r)}(0 \leq i<r)$. In general, the derivation of the reverse converter for the CF-II can be obtained as:

$X=(T \| A+B+\omega) \| x_{1}$

where for $m_{4}=2^{r}-1, A, B$, and $\omega$ equal $I, \Delta_{1}^{r} \| \bar{T}$, and 1 , respectively, and for $m_{4}=2^{r}+1, A, B$, and $\omega$ are equal to $\Gamma, T$ and $I_{(r)}$, respectively. In (4), (5), (6), (9), and (10), each of $S$ and $C$ pairs are obtained via modulo CSAs corresponding to its modulus. Moreover, each modulo $2^{\eta}-1$ adder provides a unique representation for zero.

\section{B. Proposed Hardware Architecture for Integrating the Reverse Converter into RNS Datapath}

The proposed architecture reuses the modulo adders of the datapath for integrating the reverse converter into the RNS arithmetic unit to reduce the overhead associated with the conversion from residue to two's-complement representation. The existence of common blocks between the reverse converter and datapath units in the proposed architecture makes operational segregation necessary. This process is carried out using the control signal $e$. Here, $e=0$ and $e=1$ are considered for activating the datapath and the reverse converter, respectively. Using the control signal $e$, firstly, the ports of these shared blocks are configured via multiplexers. Therefore, proper data are fed into the inputs of shared blocks depending on the operating mode of the RNS. Secondly, since the logical 


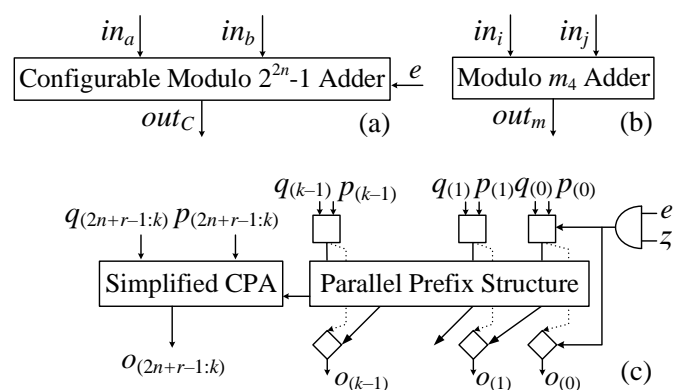

Fig. 1. The configurable integration of the reverse converter and the RNS datapath for $\left\{2^{k}, 2^{n}-1,2^{n}+1, m_{4}\right\}$ RNS.

operations of some of these blocks are different between these two modes, the required manipulation in the structure of some adders of the datapath is provided. The overall architecture of the configurable integration of the reverse converter and the RNS datapath for Class-II RNS is given in Fig. 1, In the following, the proposed architecture is described in detail.

From (5) and (13), the reverse converter architecture of the Class-II RNS, apart from the considered CF in their realization, generally require a modulo $2^{2 n}-1$ adder, a modulo $m_{4}$ adder, and a binary adder. One way to realize the required modulo $2^{2 n}-1$ adder of the Class-II RNS reverse converters is by adopting the presented method in [9]. With the approach from [9], modulo $2^{k}, 2^{n}-1$, and $2^{n}+1$ adders are employed, and the modulo $m_{4}$ adder of the datapath is the only available adder for reusing to implement the remaining adders of the reverse converter. To refine the hardware reusing and enhancing the obtained gain via integrating the reverse converter into the Class-II RNS datapath, we propose an innovative approach to realize a configurable modulo $2^{2 n}-1$ adder with employing only the modulo $2^{n}-1$ and $2^{n}+1$ adders of RNS datapath. This approach which is detailed in the next subsection provides more resources for reusing with the rest of the reverse converter adders.

By adopting the proposed method to realize the modulo $2^{2 n}$ 1 adder for computing $Y$ and $T$ in (5) and (13), respectively, the modulo $2^{n}-1$ and the modulo $2^{n}+1$ adders of the RNS datapath have been used (Fig.1 (a)). For implementing the remaining required adders of (5) and (13) (i.e. the modulo $m_{4}$ adder, and the binary adder) with the help of the RNS datapath, two of four adders of datapath ( $m_{1}$ and $m_{4}$ channel adders) are available. For implementing the required signals $L$ and $I$ in the final addition of CF-I and CF-II, respectively, the modulo $m_{4}$ adder can be employed ( $\bar{L}$ in (5) and $A$ in (13) are directly derived from $L$ and $I$, respectively, with simple logical manipulations). Since the modulo $m_{4}$ adder in the reverse conversion and the RNS datapath perform the same operation on their corresponding inputs, therefore, no manipulation is involved in this block (Fig.1 (b)). To conduct the final addition of $L\left\|Y+\Delta_{1}^{2 n}\right\| \bar{L}+1$ and $T \| A+B+\omega$ in (5) and (13), respectively, the modulo $2^{k}$ adder is the last available adder from the RNS datapath that can be exploited for hardware reusing. The final additions can be realized via two sub-circuits. The first sub-circuit employs the modulo $2^{k}$ adder (with carry-input) of the RNS datapath for the addition of $k$-least significant bits $L\left\|Y+\Delta_{1}^{2 n}\right\| \bar{L}+1$ and $T \| A+B+\omega$ with 1 and $\omega$ as carry-input, respectively. Since the functionality of the modulo $2^{k}$ adder in the datapath and the reverse converter has a minor difference, a trivial manipulation on it by employing extra AND gate should be carried out (see Fig. 1(c)). When the datapath is activated $(e=0)$, the output of the supplementary AND gate that is fed the carry input of the modulo $2^{k}$ adder equals to zero, and the adder performs the expected operation (addition modulo $2^{k}$ ). When the reverse converter is activated $(e=1), z$ signal (which equals to 1 , and $\omega$ for the first and second CFs, respectively) is fed to carry input of the $k$-bit adder, and the expected addition for the $k$-least significant bits of the final binary addition of reverse conversion is conducted. The $G_{(k-1: 0)}$ signal of the modulo $2^{k}$ adder provides the carry input of the second sub-circuit that conducts the addition of remaining bits via a cost-efficient block that does not belong to the RNS datapath. To reduce this dependency and avoiding the performance degradation of the reverse converter, for connecting these two sub-circuits a fast carry processing technique can also be adopted for the second sub-circuit (in this brief, fast carry processing refer to a technique that adds an extra level of prefix operator after the parallel prefix structure for accommodating the input carry). The second sub-circuit of the final addition is a simplified Carry Propagate Adder (CPA) due to the presence of a constant vector (all zero or all one vector) in one of the addition's operands. The weighted number $X$ is obtained by concatenating the output of the proposed architecture and $x_{1}\left(X=O \| x_{1}\right)$. For the sake of readability, only the outputs of the required multiplexers for RNS operational segregation are sketched in Fig. 1. (i.e. $i n_{a}, i n_{b}, i n_{i}, i n_{j}, p$, and $q$ ). Let $\Phi$ and $\Psi$ be the first and second operands of the final addition of the reverse converter. So, $\Phi$ and $\Psi$ for the CF-I equal to $L \| Y$ and $\Delta_{1}^{2 n} \| \bar{L}$, respectively, and for the CF-II, $\Phi$ and $\Psi$ are equal to $T \| A$ and $B$, respectively. Table I summarizes the port configurations of the proposed configurable architecture considering the value of $e$ via a multiplexing approach.

TABLE I

The Port CONFIGURATIONS OF THE PROPOSED ARCHITECTURE

\begin{tabular}{cccc}
\hline \hline \multirow{2}{*}{ Port } & \multicolumn{3}{c}{ Corresponding Values } \\
& $e: 0$ & $e: 1(\mathrm{CF}-\mathrm{I})$ & $e: 1(\mathrm{CF}-\mathrm{II})$ \\
\hline$i n_{a(n-1: 0)}$ & $x_{2}$ & $S_{Y(n-1: 0)}$ & $S_{T(n-1: 0)}$ \\
$i n_{a(2 n-1: n)}$ & $x_{3}^{\prime}$ & $S_{Y(2 n-1: n)}$ & $S_{T(2 n-1: n)}$ \\
$i n_{b(n-1: 0)}$ & $y_{2}$ & $C_{Y(n-1: 0)}$ & $C_{T,(n-1: 0)}$ \\
$i n_{b(2 n-1: n)}$ & $y_{3}^{\prime}$ & $C_{Y(2 n-1: n)}$ & $C_{T(2 n-1: n)}$ \\
$i n_{i}$ & $x_{4}$ & $S_{L}$ & $S_{I}$ \\
$i n_{j}$ & $y_{4}$ & $C_{L}$ & $C_{I}$ \\
$p_{(k-1: 0)}$ & $x_{1}$ & $\Phi_{(k-1: 0)}$ & $\Phi_{(k-1: 0)}$ \\
$q_{(k-1: 0)}$ & $y_{1}$ & $\Psi_{(k-1: 0)}$ & $\Psi_{(k-1: 0)}$ \\
\hline
\end{tabular}

\section{Proposed Configurable modulo $2^{2 n}-1$ adder}

By adopting the select-parallel-prefix block technique [11], an effective method for implementing modulo $2^{\eta}-1$ and $2^{\mu}+1$ adders by use of multiple two's-complement parallel prefix adders with fast carry processing presented in [12]. With involving some modifications, we exploit the benefits provided by the select-parallel-prefix block technique to realize the modulo $2^{2 n}-1$ adder. In contrast to [12], to realize a modulo $2^{2 n}$ 1 adder, only modulo adders of the RNS datapath are available, and also handling the single representation of zero is necessary. 
Bearing in mind that the sum of the modulo $2^{n}-1$, and $2^{n}+1$ adders bit length is equal to modulo $2^{2 n}-1$ adder bit length, the most straightforward approach to cost-effective hardware reuse is to exploit the modulo $2^{n}-1$, and $2^{n}+1$ adders of the RNS datapath. Considering the modulo $2^{n}-1$, and $2^{n}+1$ adders in regular parallel prefix (RPP) structure [13], their only difference with two's-complement adder with fast carry processing is in the applied signal to the carry input. Let $\alpha_{i}$ be the signal that is fed back to the carry input to accommodate the end-around carry for modulo $m_{2}$ and $m_{3}$ adders. For modulo $m_{2}=2^{n}-1$ adder, $\alpha_{2}$ equals to $P_{m_{2}(n-1: 0)} \vee G_{m_{2}(n-1: 0)}$ and provides proper operation of the adder with single zero representation [13]. In this brief, the considered modulo $2^{n}+1$ adders of datapath are in the diminished-1 (D1) encoding with the ability to handle zero. It should be noted that the residue $r$ in D1 encoding can be represented via the pair $\rho:\left(z_{r}, r\right)$, where for $r=0, \rho:(0,0)$, and otherwise $\rho:(1, r-1)$. According to this one to one correspondence and considering the presented method in [14], for modulo $m_{3}=2^{n}+1$ adder, $\alpha_{3}$ equals to $z_{x_{3}} z_{y_{3}} \bar{G}_{m_{3}(n-1: 0)}$ and $z_{d_{3}}=\left(z_{x_{3}} \vee z_{y_{3}}\right) \overline{\left(z_{x_{3}} z_{y_{3}} P_{m_{3}(n-1: 0)}\right)}$ is the zero indicator of the result of D1 addition, where, $z_{x_{3}}$ and $z_{y_{3}}$ are the zero indicator bits of the modulo $m_{3}$ adder input operands in the D1 encoding $\left(d_{i}=x_{i}+y_{i} \bmod m_{i}\right)$.

To obtain a programmable design with the ability to switch operation between the datapath and reverse converter, minor manipulation over the structure of modulo $2^{n}-1$, and $2^{n}+1$ adders should be carried out. Let $\varphi_{i}$ be the signal that fed to the carry input of modulo $m_{i}$ adder instead of $\alpha_{i}$ after manipulating the datapath. when the datapath $(e=0)$ is activated, $\varphi_{i}$ should take the value of $\alpha_{i}$ for accomplishing the end-around carry, and when the reverse converter $(e=1)$ is activated, $\varphi_{i}$ should take the value of $\beta_{i}$, where $\beta_{i}$ is the signal that fed to the carry input of $i$ th adder according to the presented method in [12] to construct the modulo $2^{2 n}-1$ adder. From the above discussion, the following holds:

$\varphi_{i}=\alpha_{i} \bar{e} \vee \beta_{i} e$

Since the modulo $2^{2 n}$ - 1 adder should handle the single zero representation, a more revision on the description of $\beta_{i}$ should be conducted compared to the presented method in [12]. Unlike [12] which considers $\left(A+B+c_{i n}\right) \bmod 2^{2 n}$ for modulo $2^{2 n}-1$ addition, in this brief to provide a single representation of zero, $(A+B+\Omega) \bmod 2^{2 n}$ is adopted for formulating the addition, where $\Omega$ equals one if $A+B \geq 2^{2 n}-1$. This condition is satisfied if either $A+B>2^{2 n}-1$ or $A+B=2^{2 n}-1$. The former is equivalent to $c_{\text {out }}=1$ and the latter corresponds to $P_{(2 n-1: 0)}=$ $P_{m_{3}(n-1: 0)} P_{m_{2}(n-1: 0)}$, where $c_{\text {out }}$ is the carry output of the $A$ and $B$ addition according to the presented method in [12]. As a result, considering the single representation of zero, $\beta_{2}$ and $\beta_{3}$ equal to $G_{m_{3}(n-1: 0)} \vee P_{m_{3}(n-1: 0)} \Omega_{2}$ and $G_{m_{2}(n-1: 0)} \vee P_{m_{2}(n-1: 0)} \Omega_{3}$, respectively, where $\Omega_{i}=G_{m_{i}(n-1: 0)} \vee P_{m_{i}(n-1: 0)}$. The configurable architecture to integrate the required modulo $2^{2 n}-1$ adder of the reverse converter into the RNS datapath for Class-II RNS is illustrated in Fig. 2. When the datapath is activated $(e=0)$, the parallel prefix structure's inputs $u_{(n-1: 0)}, v_{(n-1: 0)}, h_{(n-1: 0)}, f_{(n-1: 0)}$ triggered by $x_{2}, y_{2}, x_{3}^{\prime}, y_{3}^{\prime}$ signals via multiplexers, respectively. In this state, $o$ and $t$ vectors produce the expected values of $d_{2}$ and $d_{3}^{\prime}$, respectively. During the time that the reverse conversion is activated $(e=1)$, the parallel prefix structure's inputs $u_{(n-1: 0)}, v_{(n-}$ 1:0), $h_{(n-1: 0)}, f_{(n-1: 0)}$ set to $S_{(n-1: 0)}, C_{(n-1: 0)}, S_{(2 n-1: n)}, C_{(2 n-1: n)}$ via multiplexers, respectively, where $S$ and $C$ are $2 n$-bit vectors that are fed into the modulo $2^{2 n}-1$ adder of the reverse converter.

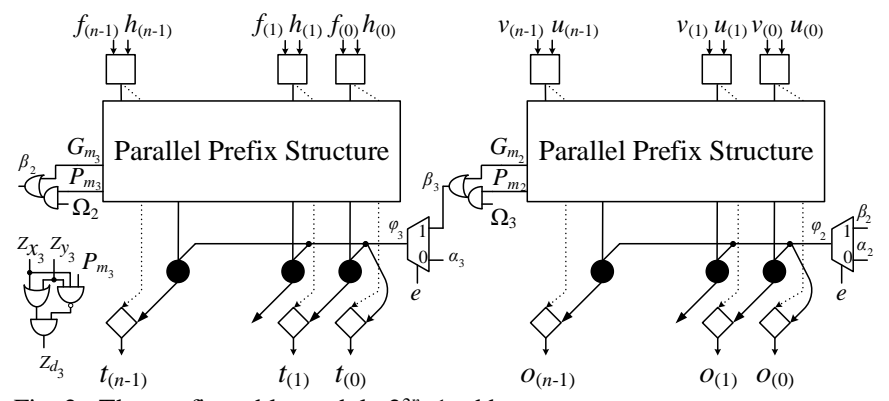

Fig. 2. The configurable modulo $2^{2 n}-1$ adder.

\section{Performance Evaluation}

To demonstrate the potential advantages of the proposed datapath/reverse-converter integration technique, we focused on the special Class-II moduli set $\left\{2^{n}, 2^{n}-1,2^{n}+1,2^{2 n+1}-1\right\}$ reverse converter. To avoid the utilization of the D1-to-normal converter block, the approach that embeds the D1-to-normal conversion into the reverse converter is adopted in this brief 0 . For investigating the impact of the proposed approach, similar to [9] our comparison contains two architectures for each of $\mathrm{CFs}$. The baseline architecture is considered the datapath of the targeted moduli set followed by its reverse converter, and the proposed architecture is deliberated the proposed design approach which embeds the reverse conversion into the RNS datapath. The area and delay of these architectures for moduli set $\left\{2^{n}, 2^{n}-1,2^{n}+1,2^{2 n+1}-1\right\}$ in CFs I and II based on the unit gate model [13] are estimated and compiled in Table II. These entries confirm significant hardware saving achieved by adopting the proposed design approach at the cost of a slight penalty in delay.

For drawing a more accurate performance comparison, the proposed architecture, along with the baseline architecture for moduli set $\left\{2^{n}, 2^{n}-1,2^{n}+1,2^{2 n+1}-1\right\}$ in two CFs I and II are described in Verilog HDL, and their functionality was verified thoroughly by ModelSim. The corresponding Verilog descriptions were synthesized with Synopsys Design Compiler through the UMC $65 \mathrm{~nm}$ CMOS technology node. To obtain the minimum achievable delay, each design is optimized for speed, by imposing increasingly stringent timing constraint during the synthesis process. From Table III, on average across all the CFs and dynamic ranges, the proposed method considerably leads to a design with smaller hardware costs compare with the

TABLE II

ESTIMATIONS OF AREA AND DELAY BASED ON UNIT GATE MODEL

\begin{tabular}{cccc}
\hline Design & Architecture & Area & Delay \\
\hline \multirow{2}{*}{ CF-I } & Baseline & $(17.5 n+1.5) \log n+108 n+17$ & $8 \log n+42$ \\
\cline { 2 - 4 } & Proposed & $(10.5 n+1.5) \log n+90 n+25.5$ & $8 \log n+54$ \\
\hline \multirow{2}{*}{ CF-II } & Baseline & $(16.5 n+1.5) \log n+126 n+15.5$ & $8 \log n+40$ \\
\cline { 2 - 4 } & Proposed & $9 n \log n+108 n+24$ & $8 \log n+52$ \\
\hline
\end{tabular}


TABLE III

PERFormanCE Figures OF THE PROPOSED, AND THE BASELINE ARCHITECTURES FOR THE MODULi SET $\left\{2^{N}, 2^{N}-1,2^{N}+1,2^{2 N+1}-1\right\}$.

\begin{tabular}{|c|c|c|c|c|c|c|c|c|}
\hline Design & $n$ & Architecture & $\operatorname{Delay}(n s)$ & Dynamic Power $(\mu W)$ & Leakage Power $(\mu W)$ & $\operatorname{Area}\left(\mu m^{2}\right)$ & $\operatorname{PDP}(f J)$ & $\operatorname{ADP}\left(\mu m^{2} . n s\right)$ \\
\hline \multirow{4}{*}{ CF-I } & \multirow{2}{*}{8} & Baseline & 0.91 & 2957.61 & 124.43 & 7141.68 & 2804.66 & 6498.93 \\
\hline & & Proposed & 0.98 & 2638.40 & 98.00 & 5877.72 & 2681.67 & 5760.16 \\
\hline & \multirow{2}{*}{16} & Baseline & 1.11 & 5260.57 & 215.09 & 12931.92 & 6077.98 & 14354.43 \\
\hline & & Proposed & 1.18 & 4636.70 & 175.59 & 10706.40 & 5678.51 & 12633.55 \\
\hline \multirow{4}{*}{ CF-II } & \multirow{2}{*}{8} & Baseline & 0.89 & 3174.31 & 124.96 & 7367.76 & 2936.35 & 6557.31 \\
\hline & & Proposed & 0.96 & 2677.20 & 105.07 & 6080.04 & 2670.98 & 5836.84 \\
\hline & \multirow{2}{*}{16} & Baseline & 1.10 & 5712.57 & 224.35 & 13437.00 & 6530.61 & 14780.70 \\
\hline & & Proposed & 1.18 & 4901.40 & 187.73 & 11121.48 & 6005.17 & 13123.35 \\
\hline
\end{tabular}

traditional design approach with a substantial $17.4 \%$ area saving, and dissipates $13.12 \%$ lower dynamic power and $17.83 \%$ lower leakage power. The area improvements can be easily explained as the hardware overhead of a given RNS is considerably reduced via the elegant hardware sharing between datapath and reverse converter. Also, the main reason behind the improvement in dynamic power dissipation as well as leakage power is fewer logic gates utilization in a given RNS through adopting the proposed design approach. The negligible penalty in delay which is $6.78 \%$ on average stemmed from the existence of extra multiplexers in the critical path and some minor modifications which are imposed to the circuit to provide switching ability between RNS datapath and reverse converter. As it can be observed in Table III, the obtained experimental results for area-delay product (ADP) and power-delay product (PDP) indicate that the proposed design approach wins the trade-off between delay and other figures of merit.

In order to evaluate the performance of the proposed design approach in a target application, RNS-based implementation of a sequential FIR filter is developed. It should be noted that since the forward converter is the same in both architectures, it is not taken into account in our evaluation. The synthesis results for a target frequency of $500 \mathrm{MHz}$ in both CFs for moduli set $\left\{2^{n}, 2^{n}\right.$ $\left.1,2^{n}+1,2^{2 n+1}-1\right\}$ with $n=8$ are compiled in Table IV. The obtained results indicate the applicability of the proposed design approach in the design of low cost and power-efficient RNS-based structures.

TABLE IV

FIGURES OF MERIT FOR THE SEQUENTIAL FIR FILTER FOR A TARGET FREQUENCY OF $500 \mathrm{MHZ}$

\begin{tabular}{cccc}
\hline Design & Architecture & $\operatorname{Area}\left(\mu m^{2}\right)$ & Power $(\mu W)$ \\
\hline \multirow{2}{*}{ CF-I } & Baseline & 13273.20 & 4521.38 \\
\cline { 2 - 4 } & Proposed & 12009.24 & 4175.74 \\
\hline \multirow{2}{*}{ CF-II } & Baseline & 13499.28 & 4738.61 \\
\cline { 2 - 4 } & Proposed & 12211.56 & 4221.60 \\
\hline
\end{tabular}

\section{CONCLUSION}

In this brief, we have presented a novel design approach to integrate the reverse converter into the datapath for Class-II RNS. Experimental evaluations showed that realizing Class-II RNS with the proposed design approach compared to the traditional design method on average occupied $17.4 \%$ lesser silicon area and reduced the dynamic power and leakage power by $13.12 \%$ and $17.83 \%$, respectively at the cost of $6.78 \%$ delay penalty. Also, the proposed design approach provided lower ADP and PDP than the traditional approach for the Class-II RNS. The obtained result also show less area and power dissipation for sequential RNS based FIR filter, when the proposed design approach is adopted. As a final conclusion, since other intermodulo operations of Class-II RNS such as comparator, sign identifier, and scaler have been the subject of a variety of studies, our work may offer an avenue in the development of these operations to refine the RNS implementation, particularly for resource-constrained devices, like those deployed on the Internet-of-Things.

\section{REFERENCES}

[1] C. H. Chang, A. S. Molahosseini, A. A. E. Zarandi and T. F. Tay, "Residue number systems: A new paradigm to datapath optimization for low-power and high-performance digital signal processing applications", IEEE Circuits Syst. Mag., vol. 15, no. 4, pp. 26-44, Nov. 2015.

[2] G. Cardarilli, L. Di Nunzio, R. Fazzolari, A. Nannarelli, M. Petricca and M. Re, "Design Space Exploration based Methodology for Residue Number System Digital Filters Implementation", IEEE Trans. Emerg. Topics Comput., pp. 1-1, 2020.

[3] L. Sousa, S. Antao and P. Martins, "Combining residue arithmetic to design efficient cryptographic circuits and systems", IEEE Circuits Syst. Mag., vol. 16, no. 4, pp. 6-32, Oct. 2016.

[4] N. Vayalil, M. Paul and Y. Kong, "A Residue Number System Hardware Design of Fast-Search Variable-Motion-Estimation Accelerator for HEVC/H.265", IEEE Trans. Circuits Syst. Video Technol., vol. 29, no. 2, pp. 572-581, 2019.

[5] N. Samimi, M. Kamal, A. Afzali-Kusha and M. Pedram, "Res-DNN: A residue number system-based DNN accelerator unit", IEEE Trans. Circuits Syst. I Reg. Papers, vol. 67, no. 2, pp. 658-671, Feb. 2020.

[6] S. Salamat, M. Imani, S. Gupta and T. Rosing, "RNSnet: In-memory neural network acceleration using residue number system", Proc. IEEE Int. Conf. Rebooting Comput., pp. 1-12, Nov. 2018.

[7] A. S. Molahosseini, A. A. E. Zarandi, P. Martins and L. Sousa, "A multifunctional unit for designing efficient RNS-based datapaths", IEEE Access, vol. 5, pp. 25972-25986, 2017.

[8] A. S. Molahosseini, L. S. de Sousa and C.-H. Chang, Embedded Systems Design with Special Arithmetic and Number Systems, New York, NY, USA:Springer, 2017.

[9] L. Sousa, R. Paludo, P. Martins and H. Pettenghi, "Towards the Integration of Reverse Converters into the RNS Channels", IEEE Trans. Comput., vol. 69, no. 3, pp. 342-348, 2020.

[10] Y. Wang, "New Chinese remainder theorems", Proc. 32nd Asilomar Conf. Signals Syst. Comput., vol. 1, pp. 165-171, Nov. 1998.

[11] A. Tyagi, "A reduced-area scheme for carry-select adders", IEEE Trans. Comput., vol. 42, no. 10, pp. 1163-1170, Oct. 1993.

[12] C. Efstathiou, H. T. Vergos and D. Nikolos, "Modulo $2^{n} \pm 1$ adder design using select-prefix blocks", IEEE Trans. Comput., vol. 52, no. 11, pp. 1399-1406, Nov. 2003.

[13] R. Zimmermann, "Binary adder architectures for cell-based VLSI and their synthesis", 1998.

[14] C. Efstathiou, H.T. Vergos, and D. Nikolos, "Handling zero in diminished-one modulo $2^{\mathrm{n}}+1$ adders", Int. J. Electronics., vol. 90, no. 2, pp. 133-144, 2003.

[15] E. Vassalos, D. Bakalis and H. T. Vergos, "Reverse converters for RNSs with diminished-one encoded channels," Eurocon 2013, Zagreb, 2013, pp. 1798-1805. 